\title{
The Fourth Law of Thermodynamics: Every Nonequilibrium State Is Characterized by a Metric in State Space with Respect to Which Its Spontaneous Attraction towards Stable Equilibrium Is along the Path of Steepest Entropy Ascent ${ }^{+}$
}

\author{
Gian Paolo Beretta \\ Brescia University, Brescia, Italy \\ + Presented at the Entropy 2021: The Scientific Tool of the 21st Century, 5-7 May 2021; Available online: \\ https://sciforum.net/conference/Entropy2021/.
}

Published: 6 May 2021

When thermodynamics is understood as the science (or art) of constructing effective models of natural phenomena by choosing a minimal level of description capable of capturing the essential features of the physical reality of interest, the scientific community has identified a set of general rules that the model must incorporate if it aspires to be consistent with the body of known experimental evidence. Some of these rules are believed to be so general that we think of them as laws of Nature, such as the great conservation principles, whose 'greatness' derives from their generality, as masterfully explained by Feynman in one of his legendary lectures. The first law and second law are universally contemplated among the great laws of Nature. In the logical development of thermodynamic theory they support the definitions of the energy and the entropy of every state of the modelled system, respectively. The recent paper https://dx.doi.org/10.1098/rsta.2019.0168 shows that in the past four decades, an enormous body of scientific research devoted to modeling the essential features of nonequilibrium natural phenomena has converged from many different directions and frameworks towards the general recognition (albeit still expressed in different but equivalent forms and language) that another rule is also indispensable and reveals another great law of Nature. We call it the 'fourth law of thermodynamics' and state it as follows: every non-equilibrium state of a system or local subsystem for which entropy is well defined, must be equipped with a metric in state space with respect to which the irreversible component of its time evolution is in the direction of steepest entropy ascent compatible with the conservation constraints. A powerful feature of the fourth law is that it provides (nonlinear) extensions of Onsager reciprocity and fluctuation-dissipation relations to the far-non-equilibrium realm.

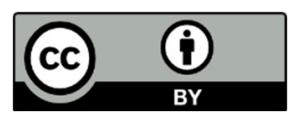

(C) 2021 by the author. Licensee MDPI, Basel, Switzerland. This article is an open access article distributed under the terms and conditions of the Creative Commons Attribution (CC BY) license (http://creativecommons.org/licenses/by/4.0/). 
